in article). Trying to answer a clinical question by a randomized clinical trial is the target at which we should always aim as researchers but most of the time this represents a prohibitive task. ${ }^{2}$ In this study the authors found that the more complex the tumor, the more likely the surgeon is forced to clamp after starting off clamp. They also found that starting the procedure off clamp with possible conversion to on clamp can be a safe plan, given that certain precautions are taken such as renal artery dissection and isolation for a quick conversion.

Unfortunately we are afraid that the debate will not end here. As in other procedures, the surgical strategy during robotic $\mathrm{PN}$ is surgeon driven. Meanwhile we should keep in mind that limiting ischemia is certainly important (especially in patients with baseline compromised renal function) but it is even more important to get a negative margin, minimize parenchymal loss and avoid complications. These are the pillars of a well performed RAPN.

William Visser and Riccardo Autorino

Division of Urology

Virginia Commonwealth University Health System

Richmond, Virginia

\title{
REFERENCES
}

1. Greco F, Autorino R, Altieri V et al: Ischemia techniques in nephron-sparing surgery: a systematic review and meta-analysis of surgical, oncological, and functional outcomes. Eur Urol 2019; 75: 477.

2. Catto JW, Blazeby JM, Holmberg L et al: In defense of randomized clinical trials in surgery: let us not forget Archie Cochrane's legacy. Eur Urol 2017; 71: 820.

\section{REPLY BY AUTHORS}

We appreciate the comments on our CLOCK trial study (reference 17 in article). We agree that the choice to change the clamping approach largely depends on surgeon attitude and experience. The transition rate in our study seems notably high at $40 \%$ but to our knowledge this information was never reported before and no comparisons with previous experiences were possible.

Nevertheless, besides surgeon related factors, 2 other reasons could explain why the off clamp approach was so frequently abandoned. 1) The permissive inclusion criteria of the study (R.E.N.A.L. score 10 or less and any diameter) allowed for randomization to off clamp PN for masses generally deemed not amenable to this approach. The risk of conversion was indeed related exclusively to tumor diameter and complexity. Others have reported that the recourse to a clampless procedure is marginal for larger masses. ${ }^{1}$ 2) Robotic systems provide enhanced vision which can be a disadvantage if bleeding occurs. With the lack of haptic feedback it may force surgeons to choose clamping to restore clean vision. Hopefully the data from the CLOCK2 trial, which is identical in design but enrolls only purely laparoscopic procedures, could contribute to test this hypothesis through an indirect comparison.

Finally, it was notable that the outcomes of converted and finalized cases overlapped, reasserting that warm ischemia is only one of the factors responsible for functional damage after $\mathrm{PN}{ }^{2}$

\section{REFERENCES}

1. Bertolo R, Autorino R, Simone $G$ et al: Outcomes of robot-assisted partial nephrectomy for clinical T2 renal tumors: a multicenter analysis (ROSULA Collaborative Group). Eur Urol 2018; 74: 226.

2. Mir MC, Autorino R and Porpiglia F: Ischemia time and beyond: the concept of global renal damage. Minerva Urol Nefrol 2018; 70: 447. 